des valeurs variant du simple au double; le jaune d'alizarine $(\mathrm{pH} 10,1$ à 12,1) s'écarte totalement des valeurs fournies par la phénolphtaléine.

Je ne parviens pas à trouver, dans tous les chiffres fournis par mes nombreux essais, une concordance un peu précise entre le degré SörenSEN et l'acidité titrée ou totale du lait et du sérum obtenu par l'alcool en présence d'une trace de chlorure de baryum. Ceci montre bien que le degré d'acidité déterminé par simple volumétrie, en présence d'un indicateur comme la phénolphtaléine, doit être une valeur conventionnelle. Je me propose de poursuivre mes recherches à ce propos.

(Université de Gand, Laboratoire de chimie alimentaire.)

\title{
VALEUR DU LAIT EN CALORIES (1)
}

\author{
par O. R. OVERMAN et F. P. SANMANN \\ Section d'Economie Laitière \\ Université dillinors, Urbana, lllinois.
}

La connaissance de la "valeur (2) du lait en calories \#, c'est-à-dire du nombre de calories qui peut être produit par la combustion complète d'une quantité déterminée de lait, est à la fois intéressante et utile à ceux qui étudient la nutrition humaine et la nutrition animale.

Des formules ont été données pour le calcul de cette valeur. GAINES et DAVIDSON (a) travaillant sur des renseignements rassemblés par STOcking et BREW (b) ont donné la formule,

$$
\mathrm{E}=49,64(2,66+t)
$$

dans laquelle $\mathrm{E}$ est la chaleur de combustion de l'extrait sec du iait et $t$ le pourcentage de matière grasse. Cette formule qui donne la valeur énergétique par livre de lait est basée sur des moyennes d'analyses; elle fut employée pour apprécier la chaleur de combustion des laits de vaches.

Fredertisen (c) indique que le Directeur A. C. Andersen donne comme chaleur de combustion du lait: pourcentage de matière grasse $x$ $113.5+290$ ealories, par kilog.

L'étude actuelle a été commencée dans le but de déterminer les chaleurs de combustion, de laits de compositions différentes, de comparer les valeurs ainsi déterminées avee les chiffres correspondants établis d'après la composition de ces laits, et de s'assurer si des formules simples pouvaient être établies par des méthodes statistiques qui donneraient des chaleurs de combustion d'une exactitude suffisante pour les usages pratiques. Ces formules seront comparées avec les formules de Gatnes et Davidson, et d'Andersen.

(1) Une étude plus complète sur ce sujet paraitra sous forme de Bulletin à la Station d'Essais Agrieoles d'Illinois.

(2) Quand il est question de calorie, c'est toujours la grande calorie qui est envisagee. 


\section{PLAN DE LA PARTIE EXPÉRIMENTALE}

Les laits étudiés furent obtenus des troupeaux d'expérience de race pure de la Section Laitière de l'Université d'Tllinois. Les échantillons représentaient des laits de vaches laitières des races Ayrshire, Guernesey, Holstein et Jersey, et de vaches du troupeau Expérimental du croisement Guernesey-Holstein.

$\mathrm{Au}$ début de l'étude, quelques échantillons furent prélevés sur de simples traites de vaches isolées. Plus tard, les échantillons furent prélevés sur les mélanges de tous les laits produits par la vache pendant. 3 jours. Les échantillons furent prélevés à tous les stades des périodes de lactation normale des vaches.

Les échantillons étaient conservés avec du formol additionné approximativement dans les proportions recommandées par PaLMER (d) et conservés dans des bocaux à fruits d'un $1 / 2$ gallon (2 litres 25 ), avec couvercle de verre et joint en caoutchouc.

Les déterminations suivantes furent faites pour tous les échantillons étudiés: poids spécifique, pourcentage de matière grasse, protéine totale, lactose, extrait sec et chaleur de combustion.

\section{PARTIE EXPÉRIMENTALE}

Le poids spécifique fut déterminé avec une balance à densité du type à chaînes. Le pourcentage de matière grasse fut déterminé par la méthode Roese-Gottuteb, en employant à peu près 5 gr. de l'échantillon.

La protéine totale fut déterminée par la méthode officielle (I) Kjeldahl.

Le lactose fut déterminé par la méthode optique officielle. Le nitrate de mercure fut employé comme précipitant. Un saccharimètre SCHMidT-HaENSCH fut employé pour toutes les polarisations.

L'extrait sec fut déterminé par la méthode officielle.

Un calorimètre adiabatique à bombe d'oxygène de Parr fut employé pour toutes les déterminations de chaleurs de combustion.

L'équivalent en eau du calorimètre fut soigneusement déterminé par plusieurs combustions de quantités pesée d'acide benzoïque pur.

Environ 10 grammes de l'échantillon de lait étaient exactement pesés dans une capsule à combustion d'Tllium, et séchés au bain-marie. Les échantillons séchés étaient conservés dans un exsiccateur jusqu'à ce que la combustion puisse avoir lieu. Les combustions avaient lieu

(1) Les méthodes officielles employées sont celles données dans l'édition de I925 des Méthodes officielles d'analyse du lait recommandées par l'Association des Chimistes Agricoles Officiels, Washington, D. C.

Voir à ce sujet la traduction française donnée par Le Lait 1925, p. 653, 769, 863, 936,1000 , et 1926 , p. $50,229$.

En ce qui concerne la méthode Kjeldahl, voir Lé Lait 1926, no 51 p. 51. 
dans l'oxygène, sous une pression de 25 à 30 atmosphères. Les changements de température étaient lus au millième de degré centigrade, sur un thermomètre BECKMANn étalonné. On faisait dans chaque cas la correction due à l'émergence de la colonne et la correction due à l'introduction du thermomètre. La chaleur de combustion totale était alors calculée en multipliant l'élévation de la température en degrés par l'équivalent en eau du calorimètre. Cette valeur était alors corrigée en raison de la chaleur dégagée par la combustion du fil de fer et de la chaleur libérée dans la formation d'acide nitrique pendant la combustion ; 212 échantillons de lait furent ainsi examinés.

Tous les résultats analytiques sont disposés en tableaux dans l'ordre croissant des pourcentages de matière grasse dans les 4 premières colonnes du tableau I. Ces résultats sont, dans chaque cas, les moyennes de doubles déterminations très proches l'une de l'autre.

\section{PARTIE STATISTIQUE (1)}

Les calories totales calculées par kilogrammes pour chaque échantillon indiqué dans la colonne 5 du tableau I furent obtenues avec une machine à calculer en exécutant les calculs indiqués ci-dessous : Calories totales par kilog $=\mathrm{A} \times 9.23+\mathrm{B} \times 5.71+\mathrm{C} \times 3.95) \times 10$

Dans cette expression, les valeurs $9.23,5.71$ et 3.95 (e) sont des grandes calories dégagées respectivement par la combustion complète d'un gramme de matière grasse de beurre, de protéine et de lactose.

Les moyennes, les déviations normales, les coefficients de corrélation et de régression partielle des résultats analytiques des colonnes A à F inclusivement, sont donnés dans le tableau II.

Les coefficients de régression partielle ont été calculés d'après la méthode indiquée par WALLACE et SNEDECOR $(\mathrm{g})$.

Des résultats donnés dans le tableau II, les équations de régression furent obtenues par substitution dans les équations de régression générale.

L'équation de régression de $\mathrm{F}$ sur $\mathrm{A}$ est :

$$
\overline{\mathrm{F}}=\mathrm{M}_{\mathrm{F}}+{ }^{\mathrm{AF}} \cdot \frac{\sigma \mathrm{F}}{\sigma \mathrm{A}}\left(\mathrm{A}-\mathrm{M}_{\mathrm{A}}\right)
$$

En substituant les valeurs données dans le tableau II et en sim plifiant, cette équation devient

$$
\overline{\mathrm{F}}=115,3277(\mathrm{~A}+2,5064)
$$

(1) Dans la partie statistique, la notation suivante est employée :

$A=$ pourcentage de malière grasse dans l'échantillon,

$\mathrm{B}=\quad$ ¿ de protéine dans l'érhantillon,

$\mathrm{C}=\quad \rightarrow$ de sucre (lactose) dans l'échantillon,

$\mathrm{F}=$ nombre de ealories déterminé par quart (=1. litre 136 environ.)

Dans cette étude, seuls la maticre grasse, l'azote et le sucre sont indiqués dans les tableaux ot utilisés dans les analyses statistiques. 
L'équation de régression pour $\mathrm{F}$ basée sur $\mathrm{A}, \mathrm{B}$ et $\mathrm{C}$ est :

$$
\begin{gathered}
\overline{\mathrm{F}}=\mathrm{M}_{\mathrm{F}}+\beta_{\mathrm{FA}} \cdot \frac{\sigma \mathrm{F}}{\sigma \mathrm{A}}\left(\mathrm{A}-\mathrm{M}_{\mathrm{A}}\right)+\beta_{\mathrm{FB}} \cdot \frac{\sigma \mathrm{F}}{{ }^{\mathrm{B}}}\left(\mathrm{B}-\mathrm{M}_{\mathrm{B}}\right)+{ }_{\mathrm{FC}} \cdot \frac{\sigma \mathrm{F}}{{ }^{\mathrm{C}}} \\
\left(\mathrm{C}-\mathrm{M}_{\mathrm{C}}\right)
\end{gathered}
$$

qui devient:

$$
\overline{\mathrm{F}_{2}}=93,39 \mathrm{~A}+54,61 \mathrm{~B}+30,60 \mathrm{C}+42.17 \quad \text { (Formule 2) }
$$

Les formules peuvent être employées pour estimer la chaleur de combustion d'un kilogramme de lait. Les valeurs ainsi calculées sont incluses dans le tableau I dans les colonnes en tête desquelles se trouvent les nombres respectifs des formules.

L'erreur normale d'estimation dans les chaleurs de combustion calculées en employant la formule 1 est:

$$
\sigma \mathrm{F} \cdot \mathrm{A}=\sigma \mathrm{F} \sqrt{1-r_{\mathrm{AF}}^{2}}=\sigma \mathrm{F} \sqrt{1-(0,9814)^{2}}=0,19119 \sigma \mathrm{F}
$$

ou $19,19 \%$ de la déviation normale de $\mathrm{F}$.

Le coefficient de corrélation multiple peut être calculée en substituant les valeurs du tableau II dans l'équation :

qui devient :

$$
\mathrm{R}^{2}=\beta_{\mathrm{FA}} \cdot{ }_{\mathrm{AF}}+\beta_{\mathrm{FB}} \cdot{ }_{\mathrm{BF}}+\beta_{\mathrm{FC}} \cdot{ }_{\mathrm{CF}}
$$

$$
\mathrm{R}^{2}=0,9834 \text { ou } \mathrm{R}=0,9917
$$

L'erreur normale d'estimation dans les chaleurs de combustion calculées par la formule 2 est :

$$
\sigma \mathrm{F} \cdot \mathrm{ABC}=\sigma \mathrm{F} \sqrt{1-\mathrm{R}^{2}}=\sigma \mathrm{F} \sqrt{1-0,9834}=0,1288 \sigma \mathrm{F}
$$

ou $12,88 \%$ de la déviation normale de $\mathrm{F}$.

Les différences entre les valeurs de la colonne $\mathrm{F}$ du tableau I et les valeurs données dans chacune des colonnes "Total ", "Formule I " et "Formule 2 " furent trouvées. Les moyennes, déviations normales et limites (1) dans la proportion de 30 sur 31 et de 100 sur 101 pour ces 3 sortes de différences sont données dans le tableau III.

\section{DISCUSSION}

Les équations de régression donnent une méthode commode à employer pour calculer la chaleur de combustion d'un quart (environ 1 litre 136) de lait si la composition du lait est connue. Si seulement A est connu, la formule 1 peut être employée. Quand A, B et C soṇt connus, la formule 2 est à employer.

(1) La déviation normale multipliée par $\pm 2,14$ donne les limites telles que les probabilités sont de 30 sur 31 pour que toute différence simple déterminée de la rnême façon tombe entre elles. Pour des probabilités de 100 sur 101, la déviation normale est à multiplier par \pm 2.58 .

Les constantes $\pm 2,14$ et $\pm 2,58$ furent déterminées d'après l'équation dorinée à la page XVIII des tables de Karl Pearson pour Statisticiens et Biologistes. 
Le tableau III, donnant les moyennes, les déviations normales et les limites dans lesquelles les résultats calculés par les formules resteront, indique la précision de leur exactitude.

La formule 1 donne des résultats qui, dans la proportion de 30 sur 31 , resteront à moins de $\pm 38,4311$ calories par $\mathrm{kg}$. de la valeur déterminée. Ces limites sont à moins d'environ $\pm 6,3 \%$ pour le lait à faible teneur en matière grasse et $\pm 3,6 \%$ pour le lait à forte teneur en matière grasse.

La formule 2 donne des résultats qui, dans la próportion de 30 sur 31, resteront à moins de $\pm 17,7061$ calories par $\mathrm{kg}$. de la valeur déterminée ou à moins d'environ $\pm 3 \%$ pour le lait à faible teneur en matière grasse et $\pm 1,7 \%$ pour le lait à forte teneur en matière grasse.

La déviation moyenne et normale des différences entre les chaleurs totales de combustion par kg., calculée et déterminée, montre qu'en ce qui concerne les valeurs pour la matière grasse, la protéine et le lactose employés dans la présente étude, les valeurs calculées sont en moyenne de 6,6505 calories par $\mathrm{kg}$. plus élevées que les valeurs déterminées. Elles montrent aussi que si 6,6505 calories sont soustraites des valeurs ealculées, il y a probabilité 30 fois sur 31 pour que de telles valeurs restent à moins de \pm 18.7175 calories, par kg. des valeurs déterminées(1).

Les erreurs normales d'estimation montrent que les écarts normaux entre les valeurs calculées et les valeurs déterminées sont: pour la formule $1: 19,19 \%$ de l'écart normal de $\mathrm{F}$ et pour la formule 2 : $12,88 \%$ de l'écart normal de $\mathrm{F}$.

Dans le but de comparer les résultats obtenus par son emploi avec les résultats obtenus en employant la formule I de la présente étude, la formule de Gaines et Davidson fut rapportée à un $\mathrm{kg}$. de lait. La formule devient alors :

$$
\text { Calories par kilog. }=109,438(2,66+t)
$$

Les résultats ealculés d'après les formules de GaInEs et Davidson, d'ANDERSEN, et la formule 1 de cette étude sont comparés graphiquement entre eux et avec les résultats moyens déterminés dans la HI. 1.

(1) Si les valeurs données comme trouvées par Andersen (c) : matière grasse de beurre: 9,11 calories : protéine de lait : 5,86 calories et lactose: 3,76 calories par gramme, sont exployées, les résultats snnt les suivants: Moyenne des différences entre les chaleurs de combustion par $\mathrm{kg}$. déterminées et les valeurs calculées par les constantes d'Andersen : 2,6561 calories; déviation moyenne de ces différences: $8,6927 \pm 2847$ calories. Ceci veut dire que la chaleur de combustion d'un $\mathrm{kg}$. de lait calculée de cette façon est en moyenne de 2,6551 calories moindre que la chaleur de combustion déterminée; 30 fois sur 31 , les límites entre lesquelies ces valeurs calculées peuvent tomber sont $\pm 18,6024$ calories, et 100 fois sur 101, les limites sont $\pm 22,4272$ calories.

Cet ouvrage d'Andersen n'était pas en notre possession quand l'étude statistique donnée dans les tableaux fut effectué. 


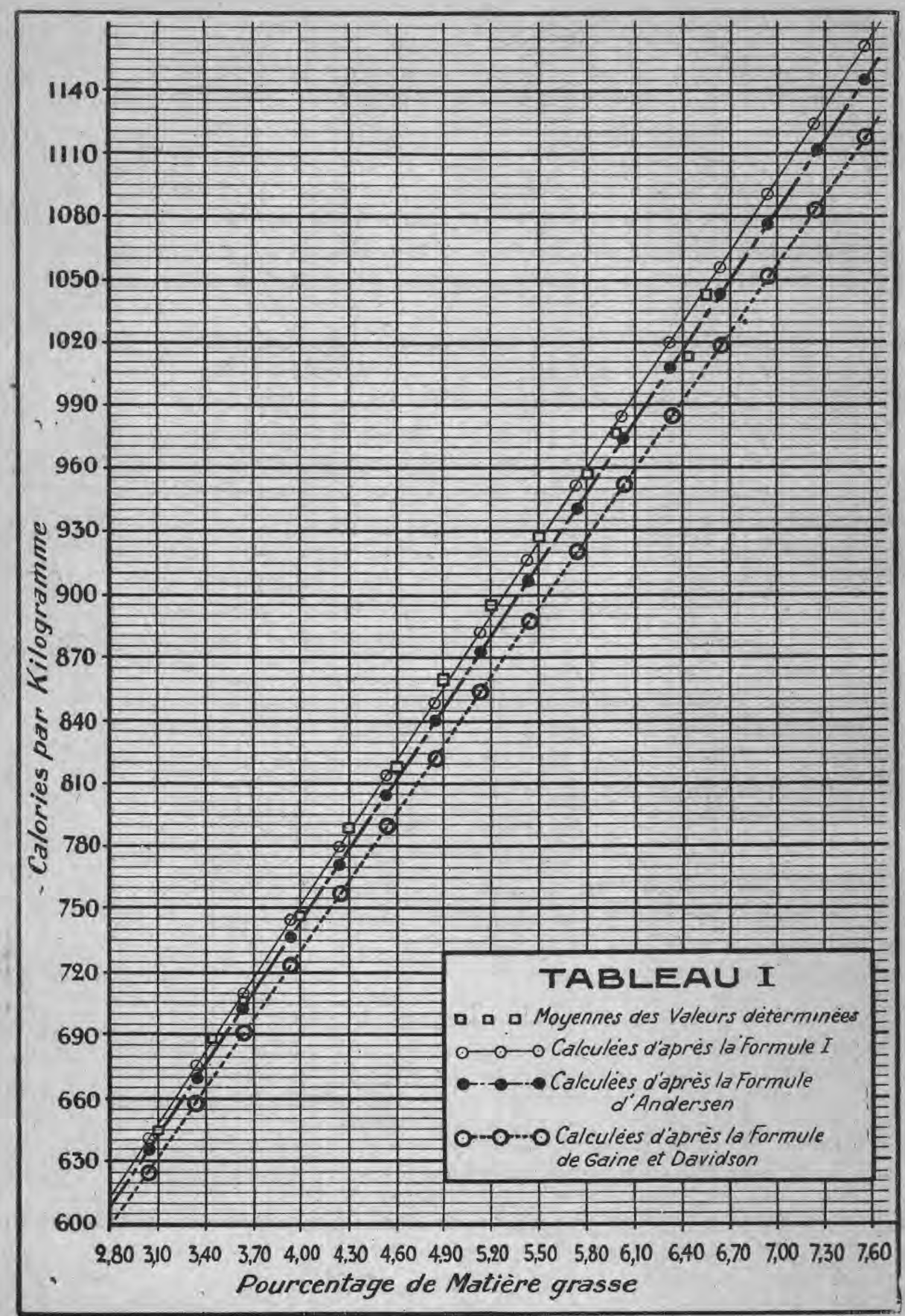


Ce graphique montre que les chaleurs de eombustion calculées par les formules coïncident très étroitement avec les valeurs déterminées. Cependant, les valeurs trouvées par la formule de GaInes et Davidson diffèrent quelque peu des valeurs déterminées quand le pourcentage de matière grasse dans les échantillons augmente.

\section{RÉSUMÉ}

L'étude de 212 échantillons de lait contenant de $2.68 \%$ à $7.59 \%$ de matière grasse a donné les résultats suivants :

1. Quand on emploie la formule (1) $\cdot \mathrm{F}=115,3277(\mathrm{~A}+2,5064)$ pour calculer la chaleur de combustion d'un $\mathrm{kg}$. de lait, on obtient des résultats tels que dans 30 cas sur 31 ils sont à moins de $\pm 38,43$ calories par $\mathrm{kg}$. de la valeur déterminée.

2. La formule $\mathrm{F}=93,39 \mathrm{~A}+54,61 \mathrm{~B}+30,60 \mathrm{C}+42.17$ donne des résultats tels que, dans 30 cas sur 31 , on obtient des résultats à moins de $\pm 17,71$ calories par $\mathrm{kg}$. de la valeur déterminée.

\section{CONCLUSIONS}

1. La chaleur de combustion d'un kg. de lait peut être calculée d'une des deux façons suivantes:

a) $\mathrm{Si}$ on ne connaît que le pourcentage de matière grasse, la formule : $\mathrm{F}=11,5,3277(\mathrm{~A}+25,064)$ doit être employée ;

b) Si lés pourcentages de matière grasse, protéine et lactose sont connus, la formule : $\mathrm{F}=93,39 \mathrm{~A}+54,61 \mathrm{~B}+30,60 \mathrm{C}+42,17$, doit être employée.

2. Les véritables chaleurs de combustion par gramme de matière grasse de beurre, de protéine de lait, de sucre de lait, 'sont probablement intermédiaires entre les valeurs données par ABDERHALDEN (e) et par Hammarsten (e) et celles données comme trouvées par ANDERSEN (c).

\section{BIBLIOGRAPHIE}

(a) Gatnes, W. L. and Davidson, F, A. : Relation between Percentage. Fat Content and Yield of Milk. IIl. Agr. Exp. Sta. Bul. 245, 1923.

(b) Stockinc, W. A. et Brew, J. D. : Mill - The Essential Food. The Dairymen's League News, Janv, 10, 1920.

(c) Frederiksen, Lars. 2e Communication de la Station d'Essais, Section d'Eeonomie Animale, Institut Royal Vétérinaire et Agricole, Copenhague (Danemark), 1925.

(d) Patmen, L. S. The preservation of Milk for Chemical Analysis. Mo. Agr. Exp. Sta. Res. Bul. 34, 29. 1919.

(e) Abderhatden, E. Chimie Physiologique, p. 333, 1908.

Hammarsten-Mandel, $"$ ( $6^{\mathrm{e}}$ édition, 1912), p. 829.

(f) Sherman, H. C. Chemistry of Food and Nutrition.

(1) Les valeurs limites sont arrondies à 2 décimales. 
(g) Wallace, H. W. et Snedecor, George W, Correlation and Machin Calculation. Ia. State Col. of Agr. and Mech. Arts. Official Publication Vol. 23, No. 35, pages 28-33.

TABLEAU 1

ANALYSE NE 212 F́CHANTILLONS DE IAAT AVEC INDICATION DES CHALEURS DE COMBUStION PAR KG. DË́terminéES ET CALCı LÉES.

\begin{tabular}{|c|c|c|c|c|c|c|}
\hline \multirow{4}{*}{ 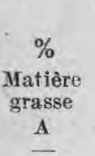 } & \multirow{4}{*}{$\begin{array}{c}\% \\
\text { Protéine } \\
\text { B }\end{array}$} & \multirow{4}{*}{$\begin{array}{c}\% \\
\text { Surre } \\
\text { C } \\
\end{array}$} & \multirow{4}{*}{$\begin{array}{l}\text { Déterminées - } \\
\text { F }\end{array}$} & \multicolumn{3}{|c|}{ Calories par Kgr. } \\
\hline & & & & \multicolumn{3}{|c|}{ Calculées } \\
\hline & & & & \multirow{2}{*}{ Par addition } & \multicolumn{2}{|c|}{ Formules } \\
\hline & & & & & I & II \\
\hline 2.68 & 2.93 & 4.72 & 595.6 & 601.1 & 598.1 & 596.9 \\
\hline 2.72 & 3.27 & 3.92 & 581.0 & 592.6 & 602.7 & 594.7 \\
\hline 2.76 & 2.86 & 4.89 & 603.9 & 611.2 & 607.4 & 605.7 \\
\hline 2.82 & 2.72 & 5.05 & 599.8 & 615.1 & 614.3 & 608.6 \\
\hline 2.85 & 2.91 & 4.81 & 605.4 & 619.2 & 617.7 & 614.4 \\
\hline 2.86 & 2.66 & 4.78 & 607.3 & 604.7 & 618.9 & 600.8 \\
\hline 2.94 & 3.10 & 4.60 & 633.8 & 630.1 & 628.1 & 626.8 \\
\hline 2.94 & 3.18 & 4.82 & 636.9 & 643.3 & 628.1 & 637.9 \\
\hline 2.94 & 2.92 & 4.46 & 622.5 & 614.3 & 628.1 & 612.8 \\
\hline 2.94 & 3.03 & 4.80 & 618.1 & 634.0 & 628.1 & 629.1 \\
\hline 2,96 & 3.06 & 4.70 & 619.5 & 633.6 & 630.4 & 629.5 \\
\hline 2.98 & 2.88 & 4.48 & 617.6 & 616.5 & 632.7 & 614.8 \\
\hline 2.98 & 3.02 & 4.84 & 621.0 & 638.7 & 632.7 & 633.5 \\
\hline 2.99 & 2.82 & 4.71 & 609.8 & 623.0 & 633.9 & 619.5 \\
\hline 2.99 & 2.94 & 4.62 & 621.1 & 626.3 & 633.9 & 623.3 \\
\hline 3.01 & 2.97 & 5.16 & 640.2 & 651.2 & 636.2 & 643.4 \\
\hline 3.02 & 2.97 & 4.75 & 638.8 & 636.0 & 637.3 & 631.7 \\
\hline 3.04 & 3,13 & 4.84 & 628.9 & 650.5 & 639.7 & 645.1 \\
\hline 3.04 & 3.16 & $5: 01$ & 653.0 & 658.9 & 639.7 & 651.9 \\
\hline 3.04 & 3.18 & 4.92 & 653.9 & 656.5 & 639.7 & 650.3 \\
\hline 3.06 & 3.15 & 5.11 & 673.3 & 664.1 & 642.0 & 656.3 \\
\hline 3.08 & 2.87 & 5.21 & 639.5 & 654.0 & 644.3 & 646.0 \\
\hline 3.09 & 2.91 & 4.96 & 645.8 & 647.3 & 645.4 & 641.4 \\
\hline 3.10 & 2.92 & 5.03 & 637.0 & 651.5 & 646.6 & 645.1 \\
\hline 3.10 & 3.00 & 4.96 & 658.5 & 653.4 & 646.6 & 647.3 \\
\hline 3.12 & 2.88 & 5.10 & 669.2 & 653.9 & 648.9 & 646.9 \\
\hline 3.12 & 3.12 & 4.93 & 648.8 & 660.9 & 648.9 & 654.8 \\
\hline 3.14 & 2.90 & 4.75 & 648.4 & 643.0 & 651.2 & 639.1 \\
\hline 3.14 & 2.91 & 4.64 & 646.6 & 639.3 & 651.2 & 636.3 \\
\hline 3.16 & 2.98 & 5.08 & 639.2 & 662.5 & 653.5 & 655.5 \\
\hline 3.17 & 3.11 & 4.48 & 651.5 & 647.1 & 654.6 & 645,1 \\
\hline 3.18 & 2.89 & 4.85 & 645.0 & 650.1 & 655.8 & 645.4 \\
\hline 3.18 & 3.03 & 5.02 & 649.8 & 664.8 & 655.8 & 658.2 \\
\hline 3.18 & 3.11 & 4.88 & 652.0 & 663.9 & 655.8 & 658.3 \\
\hline 3.19 & 3.05 & 4.74 & 649.0 & 655.8 & 657.0 & 651.7 \\
\hline 3.20 & 2.88 & 4.84 & 641.6 & 651.0 & 658.1 & 646.4 \\
\hline 3.20 & 3.26 & 4.78 & 659.7 & 670.3 & 658.1 & 665.3 \\
\hline
\end{tabular}




\begin{tabular}{|c|c|c|c|c|c|c|}
\hline \multirow{3}{*}{$\begin{array}{c}\% \\
\text { Matière } \\
\text { grasse } \\
\text { A }\end{array}$} & \multirow{3}{*}{$\begin{array}{c}\% \\
\text { Protéine } \\
\text { B }\end{array}$} & \multirow{2}{*}{$\begin{array}{c}\% \\
\text { Sucre }\end{array}$} & \multirow[b]{2}{*}{ Déterminées } & \multicolumn{3}{|c|}{ Calculées } \\
\hline & & & & Par addition & & des \\
\hline & & C & $\mathbf{F}$ & & I & II \\
\hline 3.22 & 3.00 & 4.74 & 654.4 & 657.7 & 660.4 & 651.8 \\
\hline 3.22 & 3.18 & 4.48 & 667.2 & 655.7 & 660.4 & 653.6 \\
\hline 3.25 & 2.83 & 5.18 & 661.9 & 666.2 & 663.9 & 658.7 \\
\hline 3.29 & 3.00 & 4.94 & 663.6 & 670.1 & 668.5 & 664.4 \\
\hline 3.32 & 3.09 & 5.17 & 683.5 & 687.1 & 671.9 & 679.2 \\
\hline 3.35 & 2.88 & 5.26 & 667.8 & 681.4 & 675.4 & 673.3 \\
\hline 3.37 & 3.20 & 5.03 & 687.6 & 692.5 & 677.7 & 685.6 \\
\hline 3.39 & 3.07 & 4.69 & 668.7 & $673: 4$ & 680.0 & 669.9 \\
\hline 3.41 & 2.90 & 5.24 & 685.0 & 687.3 & 682.3 & 679.3 \\
\hline 3.42 & 3.11 & 4.94 & 685.9 & 688.4 & 683.5 & 682.6 \\
\hline 3.42 & $3: 38$ & 4.83 & 699.3 & 699.4 & 683.5 & 693.9 \\
\hline 3.43 & 3.13 & 5.27 & 709.2 & 703.8 & 684.6 & 694.7 \\
\hline 3.45 & 3.06 & 4.58 & 669.3 & 674.1 & 686.9 & 671.6 \\
\hline 3.46 & 3.14 & 5.15 & 696.3 & 702.1 & 688.1 & 694.4 \\
\hline 3.47 & 3.48 & 4.77 & 700.3 & 707.4 & 689.2 & 702.2 \\
\hline 3.49 & 3.02 & 4.98 & 694.2 & 691.3 & 691.6 & 685.4 \\
\hline 3.50 & 3.10 & 4.87 & 688.5 & 692.4 & 692.7 & 687.3 \\
\hline 3.50 & 3.64 & 5.26 & 718.7 & 738.7 & 692.7 & 728.8 \\
\hline 3.51 & 2.98 & 5.14 & 692.6 & 697.2 & 693.9 & 690.0 \\
\hline 3.52 & 2.90 & 5.26 & 686.1 & 698.3 & 695.0 & 690.2 \\
\hline 3.52 & 3.01 & 4.86 & 691.4 & 688.7 & 695.0 & 684.0 \\
\hline 3.52 & 3.12 & 4.90 & 688.9 & 696.6 & 695.0 & 691.2 \\
\hline 3.56 & 2.94 & 5.21 & 693.0 & 702.3 & 699.6 & 694.6 \\
\hline 3.56 & 3.55 & 5.16 & 719.0 & 735.1 & 699.6 & 726.4 \\
\hline 3.58 & 3.22 & 5.14 & 710.7 & 717.3 & 701.9 & 709.6 \\
\hline 3.59 & 3.10 & 5,39 & 719.8 & 721.3 & 703.1 & 711.7 \\
\hline 3.60 & 3.05 & 4.82 & 694.9 & 696.8 & 704.2 & 692.4 \\
\hline 3.60 & 3.08 & 5.05 & 695.3 & 707.6 & 704.2 & 701.1 \\
\hline 3.60 & 3.52 & 4.73 & 714.2 & 720.1 & 704.2 & 715.3 \\
\hline 3.61 & 3.12 & 5.31 & 713.7 & 721.1 & 705.3 & 712.2 \\
\hline 3.62 & 2.93 & 5.10 & 709.1 & 702.9 & 706.5 & 696.3 \\
\hline 3.62 & 3.25 & 5.24 & 712.7 & 726.7 & 706.5 & 718.1 \\
\hline 3.64 & 3.06 & 4.50 & 692.6 & 688.4 & 708.9 & 686.9 \\
\hline 3.64 & 3.14 & 4.83 & 689.7 & 706.1 & 708.9 & 701.4 \\
\hline 3.64 & 3.62 & 5.16 & 723.6 & 746.5 & 708.9 & 737.7 \\
\hline 3.68 & 3.08 & 4.56 & 665.1 & 695.7 & 713.5 & 693.6 \\
\hline 3.70 & 3.00 & 4.99 & 699.8 & 709.9 & 715.8 & 704.2 \\
\hline 3.70 & 3.50 & 4.81 & 728.8 & 731.4 & 715.8 & 726.0 \\
\hline 3.71 & 2.84 & 5.34 & 705.6 & 715.5 & 716.9 & 707.1 \\
\hline 3.71 & 3.23 & 4.79 & 716.0 & 716.1 & 716.9 & 711.6 \\
\hline 3.72 & 3.08 & 4.85 & 698.4 & 710.8 & 718.1 & 706.2 \\
\hline 3.72 & 3.11 & 5.02 & 710.6 & 719.2 & 718.1 & 713.0 \\
\hline 3.75 & 3.17 & 4.99 & 713.0 & 724.2 & 721.5 & 718.2 \\
\hline 3.77 & 2.99 & 4.95 & 717.3 & 714.2 & 723.8 & 709.0 \\
\hline
\end{tabular}




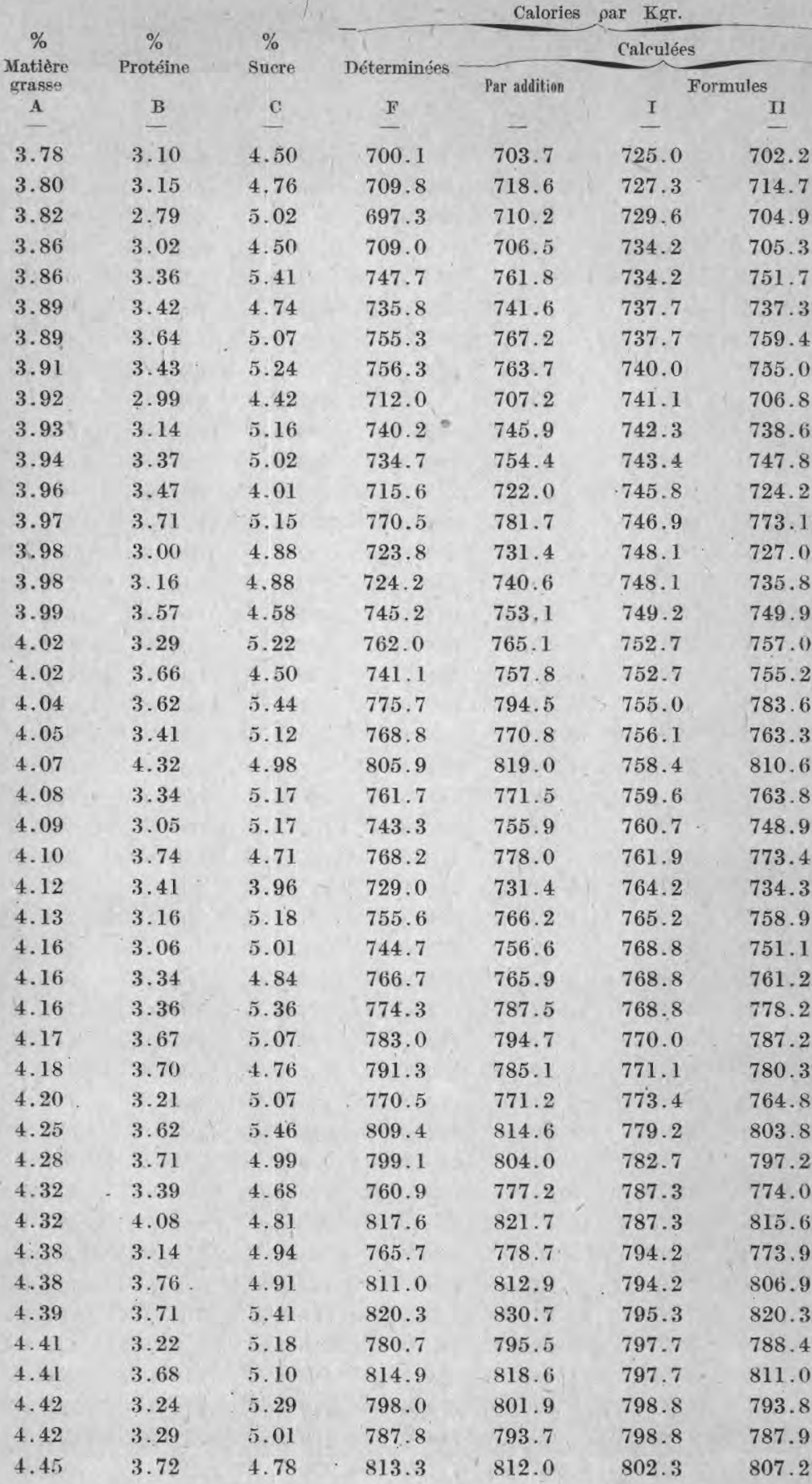


Calories par Kgr.

\begin{tabular}{|c|c|c|c|c|c|c|}
\hline \multirow{3}{*}{$\begin{array}{c}\% \\
\text { Matière } \\
\text { grasse } \\
\text { A }\end{array}$} & \multirow{3}{*}{$\begin{array}{c}\% \\
\text { Protéine } \\
\text { B }\end{array}$} & \multirow{2}{*}{$\begin{array}{c}\% \\
\text { sucre }\end{array}$} & \multirow[b]{2}{*}{ Déterminées } & \multicolumn{3}{|c|}{ Calculées } \\
\hline & & & & Par addition & & Iles \\
\hline & & C & $\mathrm{F}$ & & I & II \\
\hline & - & - & - & - & - & - \\
\hline 4.46 & 3.36 & 4.86 & 779.6 & 795.5 & 803.4 & 790.1 \\
\hline 4.46 & 3.50 & 5.06 & 810.2 & 811.4 & 803.4 & 804.7 \\
\hline 4.47 & 3.94 & 4.77 & 825.9 & 826.0 & 804.6 & 820.7 \\
\hline 4.48 & 3.48 & 5.32 & 808.7 & 822.4 & 805.7 & 813.4 \\
\hline 4.50 & 3.32 & 5.26 & 792.4 & 812.7 & 808.0 & 804.7 \\
\hline 4.52 & 3.22 & 4.41 & 775.6 & 776.3 & 810.3 & 775.1 \\
\hline 4.52 & 3.33 & 4.82 & 792.7 & 797.7 & 810.3 & 793.6 \\
\hline 4.52 & 3.40 & 5.00 & 786.3 & 808.8 & 810.3 & 803.0 \\
\hline 4.53 & 3.56 & 5.24 & 816.1 & 828.4 & 811.5 & 820.0 \\
\hline 4.55 & 3.85 & 4.92 & 821.3 & 834.1 & 813.8 & 827.9 \\
\hline 4.55 & 3.97 & 5.38 & 863.0 & 859.2 & 813.8 & 848.5 \\
\hline 4.58 & 3.80 & 4.96 & 826.2 & 835.6 & 817.3 & 829.2 \\
\hline 4.60 & 3.41 & 3.91 & 766.5 & 773.7 & 819.6 & 777.6 \\
\hline 4.62 & 2.96 & 4.60 & 787.3 & 777.1 & 821.9 & 776.0 \\
\hline 4.63 & 3.54 & 5.26 & 827.8 & 837.3 & 823.0 & 828.8 \\
\hline 4.64 & 3.36 & 5.41 & 831.4 & 833.8 & 824.2 & 824.5 \\
\hline 4.65 & 3.80 & 4.62 & 827.7 & 828.7 & 825.3 & 825.3 \\
\hline $4: 67$ & 3.68 & 3.64 & 793.2 & 784.9 & 827.6 & 790.7 \\
\hline 4.68 & 3.80 & 4.69 & 848.4 & 834.2 & 828.9 & 830.3 \\
\hline 4.74 & 4.38 & 4.72 & $876.8^{\prime}$ & 874.0 & 835.7 & 868.5 \\
\hline 4.74 & 4.04 & 5.02 & 863.4 & 866.5 & 835.7 & $859: 1$ \\
\hline 4.75 & 3.46 & 5.42 & 839.8 & 850.1 & 836.9 & 840.6 \\
\hline 4.76 & 4.08 & 5.02 & 853.5 & 870.6 & 838.0 & 863.1 \\
\hline 4.82 & 3.36 & 5.54 & 862.4 & 855.6 & 844.9 & 845.3 \\
\hline 4.87 & 3.91 & 4.59 & 863.3 & 854.1 & 850.7 & 851.0 \\
\hline 4.88 & 3.58 & 5.24 & 841.2 & 861.8 & 851.9 & 853.8 \\
\hline 4.88 & 3.70 & 5.24 & 844.7 & 868.7 & 851.9 & 860.3 \\
\hline 4.88 & 4.10 & 5.06 & 875.1 & 884.4 & 851.9 & 876.7 \\
\hline 4.94 & 3.54 & 5.59 & 860.3 & 878.9 & 858.8 & 867.9 \\
\hline 4.94 & 4.12 & 4.75 & 861.1 & 878.8 & 858.8 & 873.9 \\
\hline 4.96 & 3.23 & $\tilde{5} .08$ & 828.1 & 842.9 & 861.1 & 837.2 \\
\hline 4.96 & 3.93 & 4.88 & 879.4 & 875.0 & 861.1 & 869.3 \\
\hline 4.97 & 3.48 & 5.37 & 856.0 & 869,6 & 862.2 & 860.7 \\
\hline 4.98 & 4. 26 & 5. 08 & 892.4 & 903.6 & 863.4 & 895.3 \\
\hline 5.05 & 4.09 & 4.76 & 876.0 & 887.7 & 871.5 & 882.8 \\
\hline 5.07 & 3.84 & 4.79 & 873.5 & 876.4 & 873.8 & 871.9 \\
\hline 5.07 & 4.04 & ธ. 09 & 887.6 & 899.7 & 873.8 & 892.0 \\
\hline 5.10 & 4.01 & 4.89 & 892.3 & 892.9 & 877.2 & 887.1 \\
\hline 5.10 & 4.02 & 4,86 & 891.8 & 892.2 & 877.2 & 886.7 \\
\hline 5.11 & 4. 02 & 4.90 & 890.8 & 894.7 & 878.4 & 888.9 \\
\hline 5.19 & 4.18 & 4.82 & 901.3 & 908.1 & 887.6 & 902.6 \\
\hline 5.21 & 3.56 & 4.96 & 867.3 & 880.1 & 889.9 & 874.9 \\
\hline 5.21 & 3.59 & 5.17 & 902.5 & 890.1 & 889.9 & 883.0 \\
\hline 5.21 & 4.22 & 4.88 & 900.6 & 914.6 & 889.9 & 908.5 \\
\hline
\end{tabular}




\begin{tabular}{|c|c|c|c|c|c|c|c|}
\hline \multirow{3}{*}{$\begin{array}{c}\% \\
\text { Matière } \\
\text { grasse } \\
\text { A } \\
\end{array}$} & \multirow{2}{*}{$\begin{array}{c}\% \\
\text { Protóine }\end{array}$} & \multirow{3}{*}{$\begin{array}{c}\% \\
\text { Sucre } \\
\text { C }\end{array}$} & \multirow[b]{2}{*}{ Déterminées } & \multicolumn{3}{|c|}{ Calculées } & \\
\hline & & & & Par addition & & nules & \\
\hline & B & & $\mathbf{F}$ & -1 & I & II & \\
\hline 5.22 & 3.70 & 5.22 & 886.0 & 899.3 & 891.1 & 891.5 & \\
\hline 5.22 & 4.28 & 4.96 & 918.0 & 922.1 & 891.1 & 915.2 & \\
\hline 5.23 & 3.82 & 5.16 & 889.1 & 904.7 & 892.2 & 897.1 & \\
\hline 5.23 & 4.45 & 4.78 & 916.4 & 925.6 & 892.2 & 919.9 & \\
\hline 5.25 & 3.68 & 5.25 & 888.8 & 902.1 & 894.5 & 894.1 & \\
\hline 5.27 & 3.91 & 5.34 & 900.8 & 920.6 & 896.8 & 911.3 & b \\
\hline 5.28 & 3.66 & 4.97 & 900.5 & 892.6 & 898.0 & 887.2 & \\
\hline 5.28 & 4.07 & 5.15 & 919.7 & 923.2 & 898.0 & 915.1 & \\
\hline 5.34 & 3.45 & 5.59 & 898.4 & 910.7 & 904.9 & 900.3 & \\
\hline 5.34 & 4.13 & 4.90 & 924.1 & 922.3 & 904.9 & 916.4 & \\
\hline 5.36 & 4.24 & 4.81 & 918.9 & 926.8 & 907.2 & 921.5 & \\
\hline 5.42 & 3.95 & 5.14 & 920.2 & 928.8 & 914.1 & 921.3 & \\
\hline 5.44 & 4.06 & 5.14 & 940.1 & 937.0 & 916.4 & 929.2 & \\
\hline 5.49 & 4.78 & 4.45 & 970.1 & 955.4 & 922.2 & 952.1 & \\
\hline 5.50 & 3.71 & 5.04 & 908.5 & 918.6 & 923.4 & 912.6 & \\
\hline 5.50 & 3.79 & 5.00 & 906.7 & 921.6 & 923.4 & 915.8 & \\
\hline 5.50 & 3.90 & 5.08 & 928.9 & 931.0 & 923.4 & 924.2 & \\
\hline 5.52 & 3.90 & 5.07 & 917.6 & 932.5 & 925.7 & 925.8 & \\
\hline 5.62 & 3.87 & 4.95 & 922.9 & 935.2 & 937.2 & 929.8 & \\
\hline 5.62 & 4.21 & 4.84 & 932.1 & 950.3 & 937.2 & 945.0 & \\
\hline 5.66 & 3.62 & 5.25 & 930.2 & 936.5 & 941.8 & 929.1 & \\
\hline 5.66 & 4.21 & 5.14 & 971.4 & 965.8 & 941.8 & 957.9 & \\
\hline 5.72 & 3.83 & 5.20 & 946.7 & 952.0 & 948.7 & 944.6 & \\
\hline 5.76 & 3.52 & 4.84 & 937.3 & 923.8 & 953.3 & 920.4 & \\
\hline 5.76 & 4.08 & 5.33 & 941.9 & 975.4 & 953.3 & 966.0 & \\
\hline 5.79 & 4.00 & 5.18 & 948.7 & 967.4 & 956.8 & 959.8 & \\
\hline 5.86 & 3.73 & 4.96 & 932.1 & 949.8 & 964.9 & 944.9 & \\
\hline 5.87 & $4.19^{\circ}$ & 4.90 & 972.8 & 974.6 & 966.0 & 969.1 & \\
\hline 5.88 & 3.84 & 4.97 & 967.0 & 958.3 & 967.2 & 953.1 & \\
\hline 5.88 & 4.10 & 4.94 & 959.9 . & 972.0 & 967.2 & 966.4 & \\
\hline 5.90 & 3.98 & 5.34 & 976.8 & 982.8 & 969.5 & 973.9 & . \\
\hline 5.94 & 4.26 & 5.26 & 983.3 & 999.3 & 974.1 & 990.5 & \\
\hline 5.94 & 4.32 & 5.00 & 985.2 & 992.4 & 974.1 & 985.8 & \\
\hline 5.96 & 4.05 & 4.66 & 971.5 & 965.4 & 976.4 & 962.5 & \\
\hline 5.98 & 3.88 & 4.90 & 957.4 & 967.1 & 978.7 & 962.5 & \\
\hline 5.99 & 4.12 & 5.26 & 982.5 & 995.9 & 979.9 & 987.5 & \\
\hline 6.00 & 3.69 & 5.18 & 957.1 & 969.1 & 981.0 & 962.5 & \\
\hline 6.04 & 4.31 & 5.14 & 1001.7 & 1006.1 & 985.6 & 998.9 & \\
\hline 6.05 & $\quad 4.83$ & 4.14 & 1009.2 & 997.7 & 986.8 & 997.6 & \\
\hline 6.16 & 3.69 & 4.86 & 961.7 & 971.2 & 999.5 & 967.7 & \\
\hline 6.42 & 3.95 & 5.24 & 1013.4 & 1025.1 & 1029.5 & 1014.7 & \\
\hline 6.56 & 4.34 & 5.02 & 1042.8 & 1051.6 & 1045.6 & 1045.4 & \\
\hline 7.59 & 3.23 & 5.00 & $1096.9^{\prime}$ & 1082.5 & 1164.4 & 1080.4 & \\
\hline
\end{tabular}


TABLEAU 2

MOYENne, DÉvtation NORMALES, COEFFICIENT DE CORRÉLATION ET COEFFICIENTS DE REGRESSION PARTIELLE.

$$
\begin{aligned}
& \text { Colonne Moyenne Déviation normale } \begin{array}{c}
\text { Coefficient de } \\
\text { corrélation }
\end{array} \begin{array}{c}
\text { Coefficient de } \\
\text { régression partielle }
\end{array}
\end{aligned}
$$

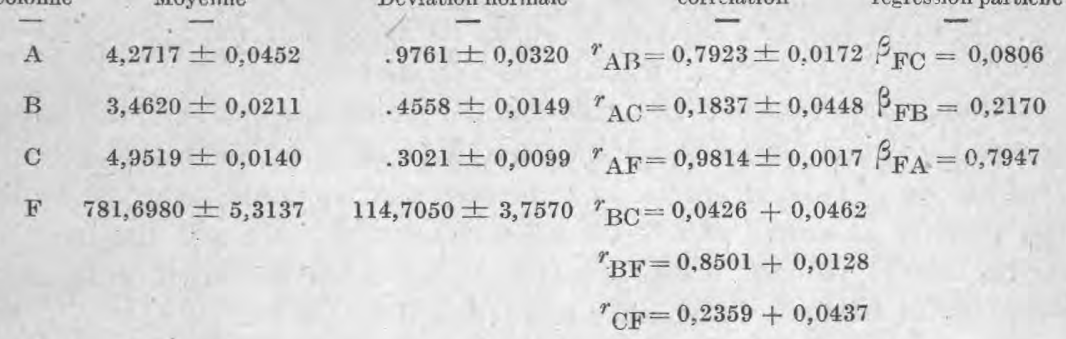

TABLEAU 3

\section{MOYENNE ET DÉVIATTON NORMALES DES DIFFÉRENCES}

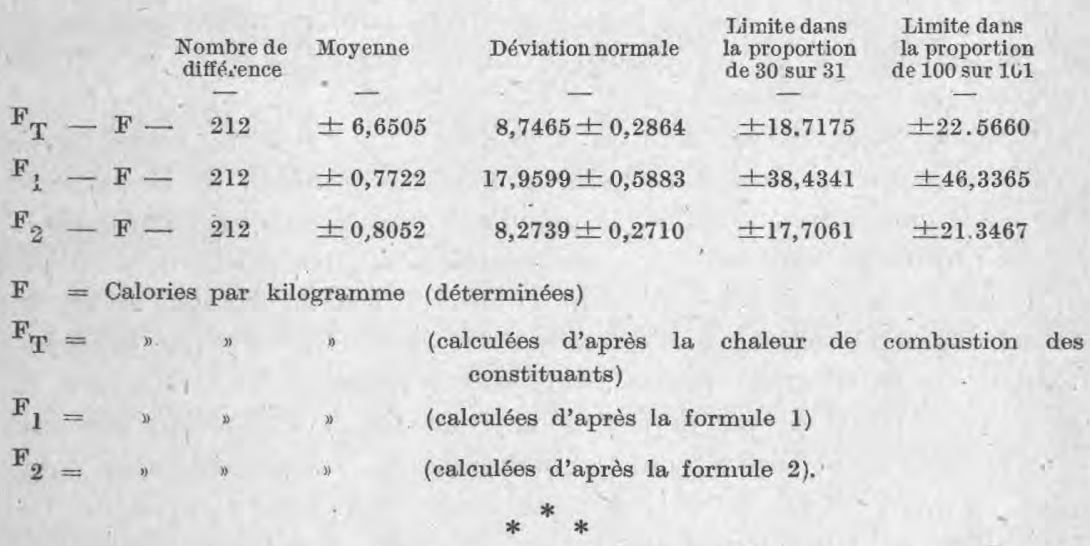

\section{NOTE DES AUTEURS}

Alors que l'article venait d'être adressé à la Revue Le Lait, les auteurs ont reçu le tirage à part d'un travail intitulé "Updersogelser over Komaelkens Sammensaetning " lu par le directeur A. C. ANDERSEN au Congrès d'Oslo, Norvège, en juin 1926.

Dans ce travail, la formule donnée pour déterminer la chaleur de combustion d'un kilog de lait est: $\mathrm{F}=300+113,50 \times$ pourcentage en matière grasse.

Les auteurs désirent relever cette notion que les résultats obtenus par l'emploi de cette formule correspondent un peu plus exactement avec ceux qui sont calculés par la formule 1 de leur travail que ne le font ceux que l'on obtient en utilisant la formule d'ANDERSEN telle qu'elle est donnée par Fredericksen. 